\title{
UNA PROPUESTA PARA EL CONTROL DE ORIENTACIÓN DE UNA TURBINA WINDFLOAT
}

\author{
Leticia Del Horno \\ 1.delhorno@upm.es \\ José A. Somolinos, \\ joseandres.somolinos@upm.es \\ Eva Segura \\ Eva.Segura@uclm.es \\ Rafael Morales2 \\ Rafael.Morales@uclm.es
}

\begin{abstract}
Resumen
La energía eólica marina debe jugar un papel importante en el cumplimiento de los compromisos ambientales asumidos en el Acuerdo de París. El uso de las soluciones flotantes para la energía eólica fuera-costa (offshore) está ganando importancia, ya que permite la expansión a un gran número de regiones en las que esto no ha sido técnicamente factible hasta ahora debido a la profundidad del lecho marino. Uno de los problemas de estas estructuras flotantes son las oscilaciones producidas por las olas y el viento, debiéndose evitar que la inclinación de la estructura supere un determinado valor durante la fase de operación. Este trabajo presenta un modelado dinámico sencillo y un sistema de control multivariable no lineal para una turbina Windfloat. Su función principal es mantener el aerogenerador en las condiciones óptimas requeridas de orientación para un mayor aprovechamiento de la energía del viento.
\end{abstract}

Palabras clave: Energía Eólica Marina; Turbinas Windfloat; Modelado Dinámico; Control no lineal

\section{INTRODUCCIÓN}

Actualmente existe un gran interés en reducir los problemas medioambientales eliminando la dependencia de los combustibles fósiles [1][2], sustituyendo éstos por energías renovables para la generación de electricidad [3][4] y, de este modo, reducir las emisiones de los gases de efecto invernadero. La Unión Europea (UE) ha establecido que más del $80 \%$ de la electricidad de la UE se produzca mediante fuentes de energía renovables para 2050, con el objetivo de lograr una reducción en las emisiones de estos gases de un $80 \%$ [5][6].
De las diversas fuentes de energía renovables disponibles, la energía eólica es hoy en día una tecnología madura, consolidada en numerosos países [7]. Además, las instalaciones offshore permiten aumentar la potencia eólica instalada [8][9] al disponer de una mayor superficie y disfrutar de algunas de las ventajas frente la energía eólica terrestre como: i) vientos más uniformes y ii) mayor velocidad debido a la ausencia de accidentes geográficos. La energía eólica marina ya lidera una gran parte de la transición energética europea. Las empresas europeas representan el $90 \%$ del mercado global offshore, cuya capacidad eólica alcanzó los 22.072 MW a finales de 2019, con 110 parques eólicos marinos en 12 países europeos y 5.047 turbinas eólicas conectadas a la red eléctrica [10-12].

Sin embargo, el costo económico asociado con su instalación y mantenimiento es alto, debido a la dificultad que implica llevar a cabo las tareas de construcción y operación en el océano. Además, los espacios apropiados en la plataforma continental son limitados y, por lo tanto, es necesario recurrir a ubicaciones lejos de la costa. Este tipo de estructuras en alta mar están unidas al fondo marino mediante líneas de amarre [13], pudiendo distinguir tres tipos: i) Boyas de mástil, ii) TLP (Tension Leg Platforms), iii) Semisumergibles. Dentro de estas últimas, el WindFloat, es el ejemplo más notable de este último tipo de estructuras [14][15].

Uno de los problemas actuales con estas estructuras eólicas marinas son las oscilaciones producidas por las olas y el viento, debiendo evitarse que la inclinación de la estructura supere los $10^{\circ}$ en la fase de operación [16][17]. Si se supera este ángulo la turbina debe desconectarse por razones de seguridad. El peligro principal no está en el riesgo de perder la estabilidad y volcar, sino en la posibilidad de que las palas golpeen a la torre en su movimiento de rotación, ya que la 
inclinación aumenta la flexión de éstas, debido a la fuerza gravitatoria y al empuje causado por el viento. En el peor de los casos podría conducirse a la destrucción de la turbina eólica, a pesar de que el eje del rotor se diseña con un ángulo de inclinación predefinido y la distancia entre la torre y las palas sea de varios metros. Por último, cuando las palas están lejos de su orientación diseñada, la eficiencia y el rendimiento de la turbina empeoran y, una carga no deseada en las palas debido al ángulo de ataque no deseado podría causar también problemas estructurales. Teniendo en cuenta estos problemas, el objetivo de este trabajo es aportar soluciones técnicas con las que reducir/eliminar sustancialmente los cambios en la orientación de las estructuras de turbinas en alta mar, a pesar de los efectos indeseables del viento y las olas. La solución propuesta se basa en el diseño y el control activo de orientación de una estructura flotante, que se consigue mediante tres columnas cilíndricas que contienen un sistema interior de lastre de agua cuya función principal es estabilizar la turbina y mantenerla vertical para mejorar su funcionamiento y, ayudar a mantener el aerogenerador marino en las condiciones óptimas necesarias para aprovechar la energía del viento.

\section{BREVE DESCRIPCIÓN DEL SISTEMA WINDFLOAT}

La figura 1 muestra la vista general CAD de la plataforma estudiada en este trabajo, que fue creada teniendo en cuenta el concepto Windfloat [18] de una turbina DTU de 10 MW [19][20]. El diseño de la plataforma se concibió con márgenes de seguridad para ser capaz de soportar las fuerzas de carga que se pueden esperar de los aerogeneradores con potencias de hasta $10 \mathrm{MW}$ en el mar. Desde la perspectiva de nuestra propuesta en cuanto al control de la orientación, los elementos más importantes del dispositivo dotan a la plataforma de su sistema de flotación, que se logra mediante tres columnas cilíndricas que contienen un sistema de lastre de agua interior cuya función principal es estabilizar la turbina y mantenerla vertical para mejorar su rendimiento. El agua dentro de los tanques de lastre se bombea internamente para compensar las importantes fuerzas y pares que actúan sobre la turbina cuando el viento interactúa con las palas. Cada uno de los tres compartimentos de lastre de agua se ubica en la mitad superior de su columna asociada. La propia turbina eólica está ubicada en la parte superior de una de las columnas, la cual proporciona flotabilidad a todo el peso de la turbina. Las otras dos columnas deben estar parcialmente provistas de lastre sólido fijo para compensar la diferencia de pesos de las tres columnas y obtener el equilibrio hidrostático que sitúa el dispositivo flotante y vertical en ausencia de cualquier tipo de perturbaciones externas. En la parte inferior de las columnas se ubican "heavy plates", que aumentan el peso y la amortiguación viscosa, debido a los vórtices que se producen en el borde de éstos. La masa añadida también aumenta debido a un mayor volumen de fluido circundante que debe moverse, lo que proporciona una mayor inercia hidrodinámica [21][22]. La plataforma se fija al fondo del mar con, al menos, tres líneas de fondeo (otros trabajos han propuesto más líneas, como [18], que propone seis).

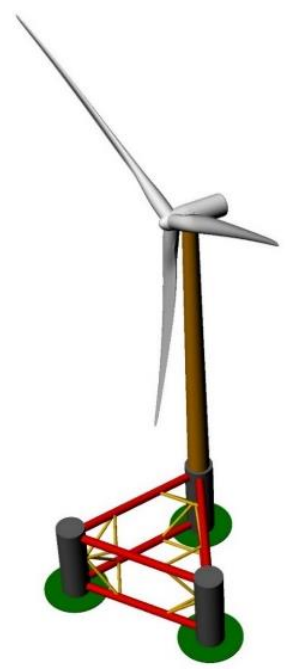

Figura 1: Vista general del sistema propuesto

\section{MODELO DINÁMICO}

La obtención de un modelo dinámico del dispositivo provisto de dos Grados de Libertad (GdL) de orientación, entra en la categoría de "Modelo de control" [23], y se considera un modelo muy simple, si no el que más. Se basa en los siguientes supuestos:

i) Sólo se consideran tres masas puntuales de idéntico valor, denotadas como $m_{i C R}$, donde el subíndice $\mathrm{i}$ indica el número de la masa (y el número de cilindros en el dispositivo como $i=1,2,3$ ) y el sub-índice CR denota "Cuerpo Rígido".

ii) No se tiene en cuenta el movimiento vertical del generador, ya que el lastre de agua se trasiega únicamente entre los tanques de lastre.

iii) Los desplazamientos del centro de gravedad son insignificantes cuando la manipulación del lastre únicamente mueve agua entre tanques.

iv) Las masas añadidas de la parte sumergida se consideran constantes, bien conocidas y son modeladas como masas puntuales.

v) La fricción viscosa se modela también como constante y se considera totalmente desacoplada entre los dos GDL.

vi) Los efectos de restauración o de computación de empuje se calculan geométricamente

vii) Sólo se tienen en cuenta pequeñas variaciones de ángulo, ya que la cuestión principal del controlador propuesto es mantener la orientación vertical del generador. 
Un nuevo sistema de referencia local $S$ definido por vectores ortogonales $\{\boldsymbol{u}, \boldsymbol{v}, \boldsymbol{w}\}$ se vincula a la turbina y se sitúa en su propio centro de gravedad (CdG). Se definen dos GdL mencionados (solo se calculan las rotaciones alrededor de los ejes $\mathrm{x}$ e y) como $\varphi_{x}$ y $\theta_{y}$, respectivamente, que permiten el sistema de referencia $S$ rotar con respecto al sistema fijo $S_{0}$. Estas variables se agrupan como un vector de coordenadas generalizadas $\mathbf{q}=\left(\begin{array}{ll}\varphi_{x} & \theta_{y}\end{array}\right)^{T}$. El sistema $S$ se sitúa únicamente desplazado una distancia vertical $z_{0}\left(\left|z_{0}\right|>\left|z_{G 0}\right|\right)$ con respecto a $S_{0}$ cuando la turbina está dispuesta con orientación nula $(\boldsymbol{q}=$ $\left.\left(\begin{array}{ll}0 & 0\end{array}\right)^{T}=\mathbf{0}\right)$, como se muestra en la Figura 2.

Una vez que el lastre fijo (basado en hormigón, balastro u otro) y los tanques de flotabilidad fijos se han ajustado para obtener un buen rendimiento de estabilidad y orientación nula, se consideran los actuadores hidrostáticos activos (una propuesta de actuadores hidrostáticos activos puede verse en [33]), los cuales serán los responsables de crear las fuerzas que accionan el dispositivo, y que se ubican idealmente en las mismas posiciones en las que se consideran colocados cada uno de los tres tanques de lastre de agua asociados a su cilindro. Estos actuadores producirán sólo fuerzas de componentes verticales las cuales se obtienen como diferencias entre las fuerzas de empuje y las fuerzas debidas a la gravedad y causadas por el trasiego del agua entre los cilindros. En aras de la simplicidad, las fuerzas de los actuadores se consideran fuerzas de empuje, lo acque está de acuerdo con la simplificación (i) que definió las masas como constantes.

Desde un punto de vista inercial, cada una de las masas puntuales de cada cilindro está formada tanto por su masa considerada rígida $m_{i C R}$ y por su masa añadida $m_{A D D_{\_} i}$, que también se considera constante:

$$
m_{i}=m_{i C R}+m_{A D D_{-} i}
$$

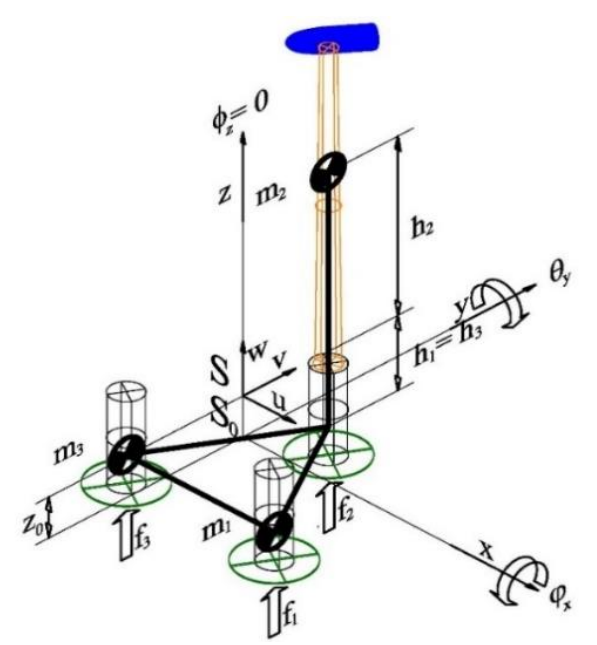

Figura 2: Sistema de referencia y magnitudes principales
La fuerza neta de cada accionamiento sobre cada uno de cilindros se obtiene calculando las siguientes fuerzas: a) la producida por los actuadores (variable de control); b) la pérdida de empuje, calculada como el volumen incremental que emerge fuera del mar debido al movimiento del dispositivo y c) la tensión incremental de las catenarias del fondeo. La i-ésima fuerza neta producida por cada actuador resulta:

$$
f_{u i}=+g \rho \Delta V_{u_{-} i}
$$

Dónde $\Delta V_{u \_i}$ denota el volumen incremental del actuador hidrostático i-ésimo, siendo ésta una variable dependiente del tiempo $\Delta V_{u_{-} i}(t)$.

\subsection{PAR DE CONTROL DE LOS ACTUADORES}

Los puntos de aplicación de las fuerzas producidas por cada uno de los actuadores o accionamientos con respecto al sistema de referencia local $S$ son:

$$
\begin{gathered}
\mathbf{P}_{1 \mathrm{~L}}=\left(\begin{array}{lll}
\frac{L}{2} & -\frac{1}{2 \sqrt{3}} L & h
\end{array}\right)^{T} \\
\mathbf{P}_{2 \mathrm{~L}}=\left(\begin{array}{lll}
0 & \frac{1}{\sqrt{3}} L & h
\end{array}\right)^{T} \\
\mathbf{P}_{3 \mathrm{~L}}=\left(\begin{array}{lll}
-\frac{L}{2} & -\frac{1}{2 \sqrt{3}} L & h
\end{array}\right)^{T}
\end{gathered}
$$

Dónde $h$ denota la componente vertical de los tanques de lastre con respecto a $S$. Cuando se producen rotaciones alrededor de los ejes fijos $\mathrm{x}$ e $\mathrm{y}$, las posiciones reales de cada uno de estos puntos con respecto a $S_{0}$ se calculan utilizando la composición de dos matrices de rotación básicas [24], obteniendo:

$$
\begin{gathered}
\mathbf{P}_{\mathrm{i}}=\mathbf{R}(\mathrm{q}) \cdot \mathbf{P}_{\mathrm{iL}} \\
\mathbf{R}(\mathrm{q})=\left(\begin{array}{ccc}
c \theta_{\mathrm{y}} & \mathrm{s} \theta_{\mathrm{y}} \mathrm{s} \varphi_{\mathrm{x}} & \mathrm{s} \theta_{\mathrm{y}} \mathrm{c} \varphi_{\mathrm{x}} \\
0 & \mathrm{c} \varphi_{\mathrm{x}} & -\mathrm{s} \varphi_{\mathrm{x}} \\
-\mathrm{s} \theta_{\mathrm{y}} & \mathrm{c} \theta_{\mathrm{y}} \mathrm{s} \varphi_{\mathrm{x}} & \mathrm{c} \theta_{\mathrm{y}} \mathrm{c} \varphi_{\mathrm{x}}
\end{array}\right)
\end{gathered}
$$

Para calcular los pares generalizados de los actuadores hidrostáticos, considerando que la transferencia de agua de lastre es interna, resulta una suma de fuerzas nula, es decir: $f_{u 1}+f_{u 2}+f_{u 3}=0$. El vector de par producido por los actuadores es:

$$
\boldsymbol{\Gamma}=\left(\begin{array}{c}
\Gamma_{\mathrm{x}} \\
\Gamma_{\mathrm{y}} \\
\Gamma_{\mathrm{z}}
\end{array}\right)=\mathbf{P}_{1} \times\left(\begin{array}{c}
0 \\
0 \\
\mathrm{f}_{\mathrm{u} 1}
\end{array}\right)+\mathbf{P}_{2} \times\left(\begin{array}{c}
0 \\
0 \\
\mathrm{f}_{\mathrm{u} 2}
\end{array}\right)+\mathbf{P}_{3} \times\left(\begin{array}{c}
0 \\
0 \\
\mathrm{f}_{\mathrm{u} 3}
\end{array}\right)
$$

Como la turbina eólica se ubica sobre la segunda columna, se considera que $f_{u 2}=-f_{u 1}-f_{u 3}$, lo cual permite obtener los pares sobre el dispositivo completo en función de únicamente dos fuerzas $f_{u 1} \mathrm{y}$ $f_{u 3}$, dando lugar a la siguiente ecuación:

$$
\boldsymbol{\Gamma}=\left(\begin{array}{c}
\Gamma_{\mathrm{x}} \\
\Gamma_{\mathrm{y}} \\
\Gamma_{\mathrm{z}}
\end{array}\right)=\left(\mathbf{P}_{1}-\mathbf{P}_{2}\right) \times\left(\begin{array}{c}
0 \\
0 \\
\mathrm{f}_{\mathrm{u} 1}
\end{array}\right)+\left(\mathbf{P}_{3}-\mathbf{P}_{2}\right) \times\left(\begin{array}{c}
0 \\
0 \\
\mathrm{f}_{\mathrm{u} 3}
\end{array}\right)
$$


Reordenando, y disponiendo los términos anteriores en forma matricial, se obtiene:

$$
\boldsymbol{\Gamma}=\frac{L}{2}\left(\begin{array}{cc}
-\sqrt{3} c \varphi_{x} & -\sqrt{3} c \varphi_{x} \\
\sqrt{3} s \varphi_{x} s \theta_{y}-c \theta_{y} & \sqrt{3} s \varphi_{x} s \theta_{y}+c \theta_{y} \\
0 & 0
\end{array}\right) \cdot\left(\begin{array}{c}
\mathrm{f}_{\mathrm{u} 1} \\
\mathrm{f}_{\mathrm{u} 3}
\end{array}\right)
$$

En la ecuación (8) se puede observar que la componente $\mathrm{z}$ del par es nulo, lo que justifica el hecho de que no es posible causar rotaciones con respecto al eje $\mathrm{z}$ con sólo fuerzas hidrostáticas verticales (Este resultado fue representado previamente en la Figura 6 como $\phi_{z}=0$ ).

Si los pares con respecto a los ejes $x$ e $y$ se agrupan como $\boldsymbol{\tau}=\left(\begin{array}{ll}\Gamma_{\mathrm{x}} & \Gamma_{\mathrm{y}}\end{array}\right)^{T}$, y fuerzas $f_{u 1}$ y $f_{u 3}$ se agrupan como $\mathbf{F}=\left(\begin{array}{ll}f_{\mathrm{u} 1} & \mathrm{f}_{\mathrm{u} 3}\end{array}\right)^{T}$, la relación final entre las fuerzas de control y los pares da como resultado la que se denomina "Matriz de Acoplamiento":

$$
\mathbf{D}(\mathbf{q})=\frac{L}{2}\left(\begin{array}{cc}
-\sqrt{3} c \varphi_{x} & -\sqrt{3} c \varphi_{x} \\
\sqrt{3} s \varphi_{x} s \theta_{y}-c \theta_{y} & \sqrt{3} s \varphi_{x} s \theta_{y}-c \theta_{y}
\end{array}\right)
$$

donde esta matriz $\mathbf{D}(\mathbf{q})$, resulta ser no diagonal en la relación de fuerzas y pares involucrados, pudiendo apreciarse que se trata de un sistema fuertemente acoplado, no lineal y dependiente del tiempo.

\subsection{PARES DEBIDOS A LA PÉRDIDA DE EMPUJE}

El volumen sumergido de cada uno de los cilindros del dispositivo no permanece constante cuando la orientación no es nula. La Figura 3 muestra un esquema simple de las columnas semisumergidas de la turbina eólica como cilindros con una rotación con un ángulo intencionalmente exagerado.

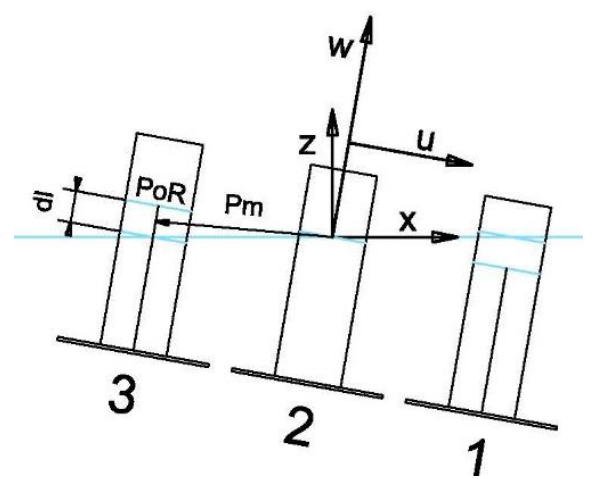

Figura 3: Esquema de la perdida de flotabilidad del cilindro 3

A partir del gráfico anterior, se obtiene fácilmente el par debido a esta pérdida de empuje:

$$
\boldsymbol{\tau}_{\text {Buoy }}=\sum_{1}^{3} \mathbf{P}_{m i} \times\left(\begin{array}{c}
0 \\
0 \\
f_{\text {Buoy_i } i}
\end{array}\right)
$$

\subsection{PAR DEL VIENTO}

Para una velocidad del viento dada, el valor de las fuerzas en el eje de la turbina eólica puede estimarse mediante el análisis la siguiente ecuación:

$$
\mathrm{F}_{w}=\frac{1}{2} C_{T} A \rho_{A i r} n^{2}
$$

donde el coeficiente $C_{T}$ es función de la relación de velocidad de la punta de pala (TSR) y de la forma geométrica de las mismas, A es el área circular cubierta por las palas de la turbina, $\rho_{\text {Air }}$ es la densidad del aire y $n$ denota la velocidad del viento.

Aunque esta fuerza debiera ser considerada como un conjunto distribuido de fuerzas sobre el rotor, a los efectos de este trabajo, puede considerar concentrada sobre el eje del rotor y su valor absoluto se denota como $F_{w}$. En notación matricial, donde $\alpha$ es el ángulo de incidencia de la fuerza sobre el eje de la góndola con respecto al plano horizontal, resulta:

$$
\mathbf{F}_{w}=\left(\begin{array}{lll}
F_{w} \cdot c \alpha & F_{w} \cdot s \alpha & 0
\end{array}\right)^{T}
$$

Pudiendo expresarse el par generalizado de los efectos del viento como:

$$
\boldsymbol{\tau}_{w}=\mathbf{R}(\mathbf{q}) \cdot\left(\begin{array}{l}
0 \\
d \\
H
\end{array}\right) \times \mathbf{F}_{w}
$$

donde las distancias $d$ y $H$ determinan la posición del eje del rotor con respecto al sistema $S$.

\subsection{DINÁMICA DE ROTACIÓN}

En esta subsección, la formulación de Lagrange permite obtener las ecuaciones de movimiento para el aerogenerador en ausencia de cualquier tipo de perturbaciones y fricciones externas. Las coordenadas generalizadas se toman como antes $\mathbf{q}=\left(\begin{array}{ll}\varphi_{x} & \theta_{y}\end{array}\right)^{T}$. Para el cálculo de la función de Lagrange, las energías cinéticas y potenciales se calculan de antemano.

\subsubsection{Matriz de inercia local}

La matriz de inercia con respecto al sistema de referencia local $S$ se obtiene de la distribución de masas puntuales propuesta. Bajo la hipótesis de simetría perfecta, $m_{1}=m_{2}=m_{3}=m$ y $m_{1}+m_{2}+$ $m_{3}=3 m$, resultando una matriz de inercia que es simétrica y definida positiva.

$\mathbf{J}=\mathrm{m}\left(\begin{array}{ccc}\frac{L^{2}}{2}+2 h_{1}^{2}+2 h_{2}^{2} & 0 & 0 \\ 0 & \frac{L^{2}}{2} 2 h_{1}^{2}+2 h_{2}^{2} & \frac{1}{\sqrt{3}} L\left(h_{1}-h_{2}\right) \\ 0 & \frac{1}{\sqrt{3}} L\left(h_{1}-h_{2}\right) & L^{2}\end{array}\right)$ 


\subsubsection{Cálculo de la energía cinética de rotación}

La energía cinética de la turbina eólica viene dada por:

$$
\mathbf{K}=\frac{\mathbf{1}}{\mathbf{2}}\left(\begin{array}{lll}
\Omega_{x} & \Omega_{y} & 0
\end{array}\right) \cdot \mathbf{R}^{T}(\mathbf{q}) \cdot \mathbf{J} \cdot \mathbf{R}(\mathbf{q}) \cdot\left(\begin{array}{c}
\Omega_{x} \\
\Omega_{y} \\
0
\end{array}\right)
$$

Donde $\Omega_{x}$ y $\Omega_{y}$ denotan las velocidades angulares sobre los ejes respectivos.

\subsubsection{Cálculo de la energía potencial de rotación}

La mencionada ausencia de cualquier tipo de perturbación, y el equilibrio hidrostático significa que la energía potencial total resulte de valor nulo.

$$
\mathbf{U}=\mathbf{0}
$$

\subsubsection{Formulación de Lagrange}

$\mathrm{Al}$ emplear las ecuaciones de Lagrange,

$$
\frac{d}{d t}\left(\frac{\partial \boldsymbol{L}}{\partial \dot{q}_{j}}\right)-\frac{\partial \boldsymbol{L}}{\partial q_{j}}=\boldsymbol{\tau}_{E X T}
$$

Siendo $\boldsymbol{L}$ la función de Lagrange, $\boldsymbol{L}=\boldsymbol{K}-\boldsymbol{U}=\boldsymbol{K}$ $j=x, y$ y denotando $\boldsymbol{\tau}_{E X T}$ como la suma de pares externos, se obtiene el modelo final.

$$
\begin{gathered}
\mathbf{M}(\boldsymbol{q}) \ddot{\boldsymbol{q}}+\mathbf{C}(\boldsymbol{q}, \dot{\boldsymbol{q}})= \\
\mathbf{D}(\mathbf{q}) \cdot \mathbf{F}-\boldsymbol{\tau}_{\text {Fric }}(\dot{\boldsymbol{q}})+\boldsymbol{\tau}_{\text {Buoy }}(\boldsymbol{q})+\boldsymbol{\tau}_{w}(\boldsymbol{q})+\boldsymbol{\tau}_{w w m}
\end{gathered}
$$

donde el par debido a los términos de fricción se aproxima empleando dos funciones cuadráticas desacopladas con coeficientes de fricción constantes según el supuesto (v) y los términos de velocidad $\dot{\boldsymbol{q}}$ :

$$
\boldsymbol{\tau}_{\text {Fric }}=\left(\begin{array}{ll}
v_{x} \varphi_{x}^{2} & v_{y} \theta_{y}^{2}
\end{array}\right)^{\boldsymbol{T}}
$$

Las variables de control en el lado derecho de la ecuación (17) fueron definidas como pares mecánicos $\boldsymbol{\tau}=\mathbf{D}(\mathbf{q}) \cdot \mathbf{F}$ a partir de las fuerzas hidrostáticas de los accionamientos según la ecuación (9). Los pares debido a la pérdida de flotabilidad $\boldsymbol{\tau}_{\text {Buoy }}$ se definieron en función de $\boldsymbol{q}$ en la ecuación (10), el par debido a la fuerza horizontal del empuje sobre el rotor $\boldsymbol{\tau}_{w}$ se definió como función de $\boldsymbol{q}$ en la ecuación (13) y, por último, se ha denotado como $\boldsymbol{\tau}_{w w m}$ los pares no modelados, los efectos del viento sobre el resto de la estructura, efectos de las olas y de las líneas de fondeo, los cuales también se consideran perturbaciones externas no modeladas.

\subsubsection{Modelo dinámico propuesto}

Agrupando todos los pares modelados, a excepción del par de control, se define $\boldsymbol{V}(\boldsymbol{q}, \dot{\boldsymbol{q}})$ como:

$$
\boldsymbol{V}(\boldsymbol{q}, \dot{\boldsymbol{q}})=\boldsymbol{C}(\boldsymbol{q}, \dot{\boldsymbol{q}})+\boldsymbol{\tau}_{\text {Fric }}(\dot{\boldsymbol{q}})-\boldsymbol{\tau}_{\text {Buoy }}(\boldsymbol{q})-\boldsymbol{\tau}_{w}(\boldsymbol{q})
$$

Pudiendo, entonces, reescribir la ecuación (18) en modo matricial de la forma:

$$
\mathbf{M}(\boldsymbol{q}) \ddot{\boldsymbol{q}}+\mathbf{V}(\boldsymbol{q}, \dot{\boldsymbol{q}})=\mathbf{D}(\mathbf{q}) \cdot \mathbf{F}+\boldsymbol{\tau}_{w w m}
$$

\section{CONTROL PROPUESTO}

La Figura 4 ilustra el esquema del sistema de control propuesto en este trabajo. Definamos una referencia deseada para ambos ejes $\mathrm{x}$ e y en forma de funciones temporales: $\varphi_{\mathrm{x}}^{*}(\mathrm{t})$ y $\theta_{\mathrm{y}}^{*}(\mathrm{t})$, respectivamente. Se emplea un regulador de realimentación no lineal para lograr el seguimiento de las variables deseadas, que está destinado a mantener su error nulo, $\boldsymbol{q}^{*}(\mathrm{t})=$ $\left(\varphi_{\mathrm{x}}^{*}(\mathrm{t}) \quad \theta_{\mathrm{y}}^{*}(\mathrm{t})\right)^{\mathrm{T}}=\mathbf{0}$, y que viene dada por (se omite la dependencia del tiempo):

$$
\boldsymbol{F}=\mathbf{D}^{-\mathbf{1}}(\mathbf{q}) \cdot\left[\mathbf{M}(\boldsymbol{q}) \boldsymbol{\Gamma}_{u}+\mathbf{V}(\boldsymbol{q}, \dot{\boldsymbol{q}})\right]
$$

Ecuación en la que la señal de control auxiliar $\boldsymbol{\Gamma}_{u}$ queda:

$\boldsymbol{\Gamma}_{u}=\ddot{\boldsymbol{q}}^{*}-\boldsymbol{K}_{D}\left[\dot{\boldsymbol{q}}-\dot{\boldsymbol{q}}^{*}\right]-\boldsymbol{K}_{P}\left[\boldsymbol{q}-\boldsymbol{q}^{*}\right]-\boldsymbol{K}_{I} \int\left[\boldsymbol{q}-\boldsymbol{q}^{*}\right] \mathrm{d} \tau$

$\boldsymbol{K}_{P}, \boldsymbol{K}_{\mathrm{I}}$ y $\boldsymbol{K}_{D}$ son matrices definidas positivas diagonales que agrupan los parámetros de un regulador multivariable de tipo PID.

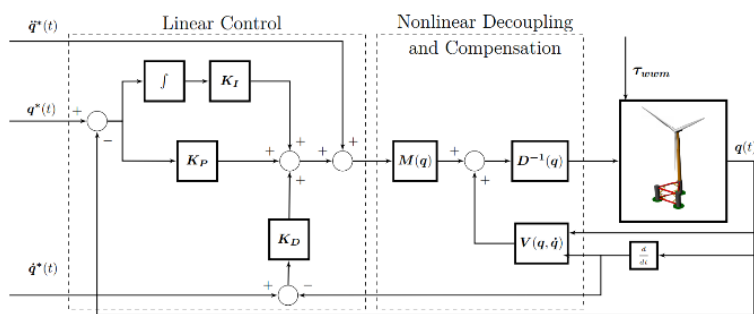

Figura 4: Esquema de control no lineal para los dos GdL Windfloat

Como se observa en la Figura 4, el esquema de control está compuesto por un bucle interno no lineal basado en el modelo dinámico propuesto y el bucle externo basado en controladores PID clásicos que se encargan de obtener la dinámica de circuito cerrado deseada del sistema controlado. El objetivo principal del bucle interno es obtener una relación entrada/salida lineal y desacoplada en ausencia de cualquier tipo de perturbaciones externas, que se denominan $\boldsymbol{\tau}_{w w m}$.

El bucle interno requiere el cálculo de la inversa de la matriz de acoplamiento $\boldsymbol{D}(\mathbf{q})$, que resulta invertible, para el rango de ángulos previsto, con la excepción de los valores de $\mathrm{c} \varphi_{\mathrm{x}} \mathrm{c} \theta_{\mathrm{y}}=0$, que son inalcanzables.

La dinámica del vector de error del bucle externo $\boldsymbol{e}_{q}=$ $\left(\boldsymbol{q}^{*}-\boldsymbol{q}\right)=\left(\begin{array}{ll}\varphi_{\mathrm{x}} & \theta_{\mathrm{y}}\end{array}\right)^{T}$ se obtiene después de sustituir las expresiones (22) y (23) en (21). En ausencia de cualquier tipo de perturbación externa:

$$
\ddot{\boldsymbol{q}}=\ddot{\boldsymbol{q}}^{*}-\boldsymbol{K}_{D}\left[\dot{\boldsymbol{q}}-\dot{\boldsymbol{q}}^{*}\right]-\boldsymbol{K}_{P}\left[\boldsymbol{q}-\boldsymbol{q}^{*}\right]-\boldsymbol{K}_{I} \int\left[\boldsymbol{q}-\boldsymbol{q}^{*}\right] \mathrm{d} \tau
$$


Este error, evoluciona gobernado por la siguiente ecuación matricial polinómica de tercer orden:

$$
\boldsymbol{e}_{\boldsymbol{q}}^{(3)}+\boldsymbol{K}_{D} \ddot{\boldsymbol{e}}_{\boldsymbol{q}}+\boldsymbol{K}_{P} \dot{\boldsymbol{e}_{\boldsymbol{q}}}+\boldsymbol{K}_{I} \boldsymbol{e}_{q}=\mathbf{0}
$$

Las matrices del regulador $\left\{\boldsymbol{K}_{D}, \boldsymbol{K}_{P}, \boldsymbol{K}_{I}\right\}$ a diseñar se agrupan de modo que representen una matriz diagonal $\mathcal{M}(s)$ definida como:

$$
\mathcal{M}(s)=I^{2 \times 2} s^{3}+K_{D} s^{2}+K_{P} s+K_{I}
$$

La dinámica de bucle cerrado deseada se propone entonces de [25][26]:

$$
\mathcal{M}^{\text {des }}(s)=I^{2 \times 2}\left(s^{3}+5 \mathrm{a}^{2}+8 \mathrm{a}^{2} s+4 \mathrm{a}^{3}\right)
$$

La cual puede definirse en función de un único parámetro de diseño $a$ (en $\mathrm{rad} / \mathrm{s}$ ). Un sistema lineal con un polinomio característico mono-variable proporcionado por la ecuación (27) tiene una respuesta de tiempo similar al conocido sistema de segundo orden con frecuencia natural $\omega_{n}=a$ y críticamente amortiguado. Las matrices de diseño $\left\{\boldsymbol{K}_{D}, \boldsymbol{K}_{P}, \boldsymbol{K}_{I}\right\}$ se obtienen, finalmente, se obtienen directamente igualando las ecuaciones (26) y (27).

\section{RESULTADOS DE LA SIMULACIÓN}

En este apartado se muestran los resultados de simulaciones del modelo dinámico implantado en OrcaFlex, así como el sistema de control descrito anteriormente para demostrar la bondad del mismo para lograr la orientación deseada a través de las fuerzas obtenidas mediante la gestión del agua de lastre.

\subsection{PARAMETRO DE DISEÑO -a-}

Pueden tomarse varios modelos ambientales para lograr simulaciones realistas de cualquier tipo de dispositivo marino. En este trabajo, se escoge un espectro de ola de Pierson-Moskowitz (PM) [27] $\mathrm{W}(\omega, \psi)$ en función de la frecuencia $\omega$ y la dirección $\psi$. Debido a la dinámica en bucle cerrado esperada para el dispositivo, debido a la presencia del modelo superpuesto de baja frecuencia (LF) y de frecuencia de olas (WF), solo los efectos de LF se considerarán como perturbaciones externas.

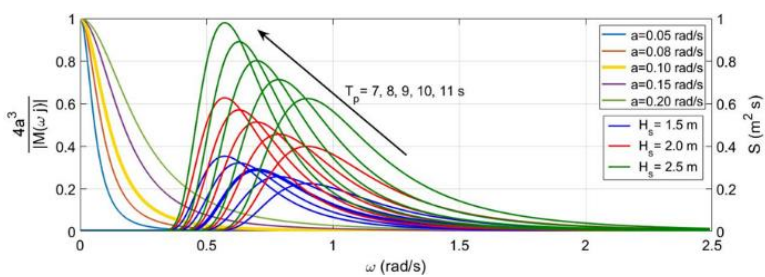

Figure 5: Parámetro de diseño
La Figura 5 muestra el espectro PM para una altura de olas significativa de $1.5,2$ y $2.5 \mathrm{~m}$ para períodos pico de $7,8,9,10,11 \mathrm{~s}$, junto con la magnitud de Bode (gráfica lineal en lugar de logarítmica para facilitar la comparación) para la dinámica deseada en bucle cerrado propuesta con parámetros de diseño a diferentes, cuyos valores son $0.05,0.075,0.1,0.15$, $0.2 \mathrm{rad} / \mathrm{s}$. Al observar el gráfico anterior, se aprecia que la interacción con las olas de la dinámica en bucle cerrado deseada con a $=0.15 \mathrm{rad} / \mathrm{s}$ y a $=0.2 \mathrm{rad} / \mathrm{s}$, es mayor que la del resto. Por otro lado, las dinámicas correspondientes a reguladores para $\mathrm{a}=0.05 \mathrm{rad} / \mathrm{s}$ y para $\mathrm{a}=0.075 \mathrm{rad} / \mathrm{s}$ se consideran demasiado lentas. Se opta, por tanto, por un parámetro de diseño de a = $0.1 \mathrm{rad} / \mathrm{s}$.

\subsection{PERTURBACIONES EXTERNAS DEL VIENTO}

Los resultados de la simulación que se presentan a continuación en la Figura 6 no representan todavía un caso realista, pero este tipo de simulaciones resultan muy útiles para verificar la bondad del controlador propuesto [26]. En esta simulación, el sistema ha sido sometido a perturbaciones del viento con transiciones en escalón tanto en velocidad, $n(t)(\mathrm{m} / \mathrm{s})$, como en orientación, $\alpha(t)\left({ }^{o}\right)$, tomando los siguientes valores en función del tiempo:

$$
\begin{aligned}
& n(t)=\left\{\begin{array}{cc}
0 & 0 \leq t<100 \\
7.5 & 100 \leq t<400 \\
15 & 400 \leq t<700 \\
10 & 700 \leq t \leq 1100
\end{array}\right. \\
& \alpha(t)=\left\{\begin{array}{cc}
0 & 0 \leq t<550 \\
22.5 & 550 \leq t<1100
\end{array}\right.
\end{aligned}
$$

La Figura 6.a muestra que la plataforma no puede mantener una orientación nula alrededor del eje $x$ cuando se encuentra en bucle abierto, alcanzando un ángulo de escora máximo de $-23^{\circ}$ después del primer escalón en velocidad del viento, además de mostrar una respuesta oscilante. Cuando se produce el segundo escalón en velocidad del viento, la oscilación de la plataforma en el eje y también aumenta. Debido a la orientación del viento inicial, el cambio en la inclinación de la plataforma alrededor del eje y en bucle abierto, (Figura 6.b), es apreciable solamente después del escalón en la dirección del viento, aumentando su ángulo de escora hasta aproximadamente $6^{\circ}$. Las respuestas en bucle cerrado ante las mismas señales se han superpuesto con las anteriores, pudiendo apreciarse cómo el sistema de control propuesto es capaz de compensar los efectos del viento, incluso ante cambios bruscos en escalón, a la vez que se percibe un muy pequeño acoplamiento en las respuestas en ambos GdL. 


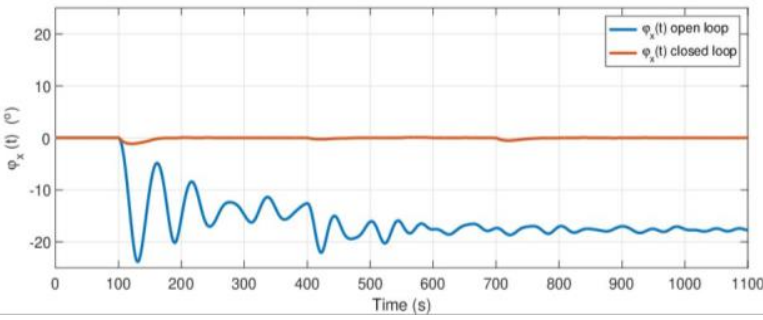

a.- Respuestas en bucle abierto y en bucle cerrado alrededor del eje $\mathrm{x}$

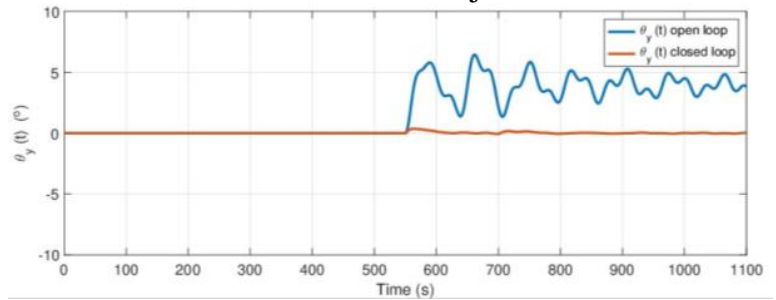

b.- Respuestas en bucle abierto y en bucle cerrado alrededor del eje y

Figura 6: Respuestas en bucle abierto y bucle cerrado bajo variaciones del viento en escalón

Este desacoplamiento no perfecto apreciable en el comportamiento de la plataforma en bucle cerrado, ante las mismas perturbaciones del viento según (28), se debe a los efectos de los términos no modelados, y por ende, no compensados. Incluso los efectos de los cambios bruscos en la velocidad y orientación del viento en el comportamiento de la plataforma son satisfactorios, mostrando en las Figuras 6.a como en la 6.b, el correcto funcionamiento del término de desacoplamiento definido en la ecuación (9).

Los siguientes resultados temporales (Figura 7) muestran las variables angulares ante la simulación de un caso más realista que el anterior. Las propiedades del modelo de viento utilizado se modelan según una distribución de Weibull, y fueron reconstruidos a partir de los datos reales registrados por la boya de $\mathrm{La}$ Langosteira II $(43.35 \mathrm{~N}, 8.56 \mathrm{~W})$ [28]. A esta señal se le han añadido efectos de las olas como perturbaciones externas, con un período de 9 s y una altura significativa de $1.5 \mathrm{~m}$, según los datos más frecuentes recogidos por la boya.

La orientación de la plataforma en bucle abierto da como resultado variaciones máximas en la inclinación de hasta $\varphi_{x}(t)=23^{\circ}$ y $\theta_{y}(t)=6^{\circ}$ cuando se encuentra sometida a la acción del viento. El único momento que está cerca del valor nulo para ambos ángulos (aunque oscila debido a las olas), es en la primera y última sección de la simulación donde la acción del viento se anula. Para la respuesta en bucle cerrado, se puede apreciar que el sistema de control implementado mantiene la verticalidad de la plataforma.

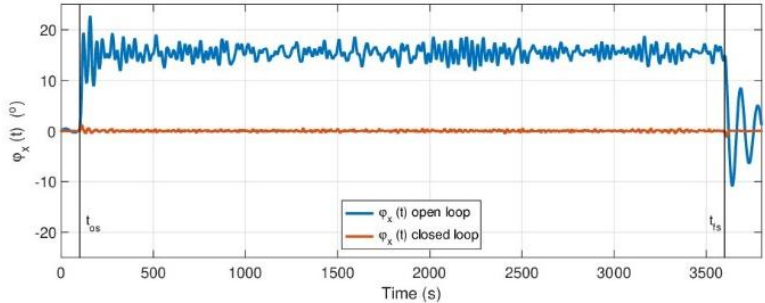

a.- Respuestas en bucle abierto y en bucle cerrado alrededor del eje $\mathrm{x}$

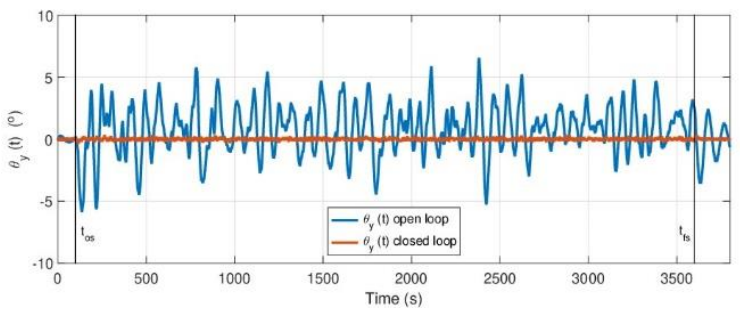

b.- Respuestas en bucle abierto y en bucle cerrado alrededor del eje y

Figura 7: Simulación completa de la respuesta de bucle abierto y bucle cerrado del dispositivo bajo datos reales de viento y oleaje tomados de [28]

Con las simulaciones anteriores se ha podido comprobar que el sistema de control propuesto es robusto para compensar parte de los efectos no modelados como los debidos a los cables de fondeo o perturbaciones externas como las olas, obteniendo una muy significativa mejora en el comportamiento de la plataforma.

\section{CONCLUSIONES}

El crecimiento demográfico en el mundo, junto con el cambio climático, subrayan la necesidad de un suministro de energía fiable y sostenible. Una solución viable a los desafíos energéticos actuales pasa por la explotación de la energía eólica marina que, en los últimos años, se ha convertido en el principal pilar de la industria de las energías renovables debido a que no tiene costes asociados a los combustibles, es una energía prácticamente libre de $\mathrm{CO}_{2}$, responde a los problemas ambientales del cambio climático y es inagotable. El uso de soluciones flotantes está ganando protagonismo en la energía eólica marina debido a que se multiplica el potencial energético de esta forma de energía varias veces al obtener un mejor uso del viento y factores de capacidad de instalación mucho más altos. Sin embargo, un problema actual en las estructuras eólicas marinas son las oscilaciones producidas por las olas y el viento, debiéndose restringir la inclinación de la estructura a ángulos menores de $10^{\circ}$ en la fase de operación. 
Este trabajo propone un nuevo modelo dinámico extraordinariamente simple y un algoritmo de control basado en modelo para turbinas Windfloat con el objetivo de reducir/eliminar sustancialmente los cambios en la orientación de la estructura flotante y obtener un aprovechamiento óptimo del viento y factores de capacidad mucho más elevados. El modelo dinámico propuesto se basa únicamente en la definición de tres masas puntuales que permiten convertir las fuerzas de los tres actuadores hidrostáticos en dos pares que son responsables de las rotaciones de la estructura Windfloat. Las principales ventajas del modelo dinámico propuesto son que resulta i) lo suficientemente preciso para describir el movimiento de la turbina Windfloat y ii) lo suficientemente simple como para ser utilizado en el diseño del algoritmo de control. Además, la concepción del sistema de control no lineal puede descomponerse en los siguientes tres términos: i) una matriz denominada de desacoplamiento que es responsable de desacoplar los movimientos de la turbina Windfloat para valores de orientación no singulares; ii) una compensación de los pares centrípetos y de Coriolis por medio de un modelo no lineal basado en el desacoplamiento, y iii) la definición de una acción de control auxiliar clásica del tipo proporcional-integral-derivada (PID). La dinámica de bucle cerrado del sistema se eligió simplemente diseñando ganancias proporcionales, integrales y derivadas, ilustrando así que el algoritmo de control propuesto es simple, computacionalmente eficiente y adecuado para su implementación en un sistema basado en computador.

Se han proporcionado resultados de simulaciones numéricas, en las que se evalúa la eficacia del método de control propuesto con respecto a las incertidumbres paramétricas (por ejemplo, el efecto de los cables de fondeo) en el sistema y las perturbaciones no modeladas. Los resultados obtenidos ilustran que la turbina Windfloat se comporta extremadamente bien con el tiempo de establecimiento deseado y sin sobreoscilación, ante perturbaciones en escalón, logrando una orientación prácticamente nula en ambos ángulos de orientación de la plataforma, con errores minúsculos de aproximadamente $0.02^{\circ}$ teóricos.

Por último, el estudio de diferentes estrategias de control que mejoren el aprovechamiento del recurso eólico y los factores de mayor capacidad serán los temas de nuestra futura investigación.

\section{Agradecimientos}

Al personal del Grupo de Investigación Tecnológico en Energías Renovables Marinas de la UPM -GITERM-.

\section{English summary}

\section{A PROPOSAL FOR THE CLOSED- LOOP ORIENTATION CONTROL OF A WINDFLOAT TURBINE SYSTEM}

\begin{abstract}
Offshore wind power is called upon to play an important role in fulfilling the environmental commitments acquired in the Paris Agreement. The use of floating solutions for offshore wind energy is gaining importance as it allows expansion to many regions where this has not been technically feasible until now due to depth. One of the problems of these floating structures is the oscillations produced by the waves and the wind, and the inclination of the structure must be prevented from exceeding a particular value during the operation phase. This work presents a simple dynamic model and a nonlinear multivariable control system for a Windfloat turbine. Its principal function is to keep the wind turbine in the optimal conditions required to use wind energy better.
\end{abstract}

Keywords: Offshore Wind Energy; Windfloat Turbines; Dynamic Modeling; Nonlinear Control.

\section{Referencias}

[1] C. Stokes et al. "Anticipated coastal impacts: What water-users think of marine renewables and why," Ocean Coast. Manag., vol. 99, no. C, 2014, doi: 10.1016/j.ocecoaman.2014.04.003.

[2] F. Dalir et al. "A dynamic quasi comprehensive model for determining the carbon footprint of fossil fuel electricity: A case study of Iran," J. Clean. Prod., Jul. 2018, doi: 10.1016/j.jclepro.2018.03.274.

[3] M. H. Amrollahi et al. "Techno-economic optimization of hybrid photovoltaic/wind generation together with energy storage system in a stand-alone micro-grid subjected to demand response," Appl. Energy, vol. 202, 2017, doi: 10.1016/j.apenergy.2017.05.116.

[4] D. Younesian and M. R. Alam, "Multi-stable mechanisms for high-efficiency and broadband ocean wave energy harvesting," Appl. Energy, 2017, doi: 10.1016/j.apenergy.2017.04.019.

[5] European Commission, "A Clean Planet for all A European strategic long-term vision for a prosperous, modern, competitive and climate neutral economy," Nov. 2018. 
[6] European Commission, "Offshore Wind Energy: Action needed to deliver on the Energy Policy Objectives for 2020 and beyond" 2008.

[7] M. Mikati et al. "Electric grid dependence on the configuration of a small-scale wind and solar power hybrid system," Renew. Energy, vol. 57, Sep. 2013, doi: 10.1016/j.renene.2013.02.018.

[8] D. Y. C. Leung et al. "Wind energy development and its environmental impact: A review," Renewable and Sustainable Energy Reviews, vol. 16, no. 1. Pergamon, 2012, doi: 10.1016/j.rser.2011.09.024.

[9] A. D. Sahin, "Progress and recent trends in wind energy," Progress in Energy and Combustion Science, vol. 30, no. 5. Elsevier Ltd, pp. 501543, 2004, doi: 10.1016/j.pecs.2004.04.001.

[10] I. Renewable Energy Agency, Wind energy: A gender perspective. 2020.

[11] W. Europe, "Offshore Wind in Europe Key trends and statistics 2019," 2020.

[12] REN21., "Renewables 2019 Global Status Report," 2019.

[13] J. Harrison et al. "Floating Offshore Wind: Installation, Operation \& Maintenance Challenges Approval / Revision History Version: A Floating Offshore Wind: Installation Challenges," 2020.

[14] L. Castro-Santos et al. "Economic feasibility of floating offshore wind farms," Energy, vol. 112, Oct. 2016, doi: 10.1016/j.energy.2016.06.135.

[15] "WindFLoat Atlantic project | edp.com." https://www.edp.com/en/innovation/windfloat (acc. Sep. 15, 2020).

[16] IEC, "Wind turbines-Part 1: Design requirements," 2005. Accessed: Oct. 13, 2020. [Online]. Available: www.iec.ch.

[17] R. Antonutti et al. "The effects of wind-induced inclination on the dynamics of semi-submersible floating wind turbines in the time domain," Renew. Energy, vol. 88, pp. 83-94, Apr. 2016, doi: 10.1016/j.renene.2015.11.020.

[18] D. Roddier et al. "WindFloat: A floating foundation for offshore wind turbines," J. Renew. Sustain. Energy, vol. 2, no. 3, p. 033104, May 2010, doi: 10.1063/1.3435339.
[19] "DTU 10MW RWT / dtu-10mw-rwt · GitLab." https://rwt.windenergy.dtu.dk/dtu10mw/dtu10mw-rwt (acc. Sep. 27, 2020).

[20] M. H. H. Christian Bak, et al. "Description of the DTU 10 MW Reference Wind Turbine", 2013.

[21] C. Lopez-Pavon et al. "Hydrodynamic coefficients and pressure loads on heave plates for semi-submersible floating offshore wind turbines: A comparative analysis using large scale models," Renew. Energy, vol. 81, Sep. 2015, doi: 10.1016/j.renene.2015.04.003.

[22] A. Lavrov et al. "Modeling the Heave Oscillations of Vertical Cylinders with Damping Plates. Safe Offloading from Floating LNG Platforms View project MARSTRUCT-Network of Excellence on Marine Structures"," 2016, doi: 10.3940/rina.ijme.2016.a3.365.

[23] T. I. Fossen, Handbook of Marine Craft Hydrodynamics and Motion Control. 2011.

[24] B. Siciliano et al. Robotics: Modelling, Planning and Control (2nd edition). 2010.

[25] M. Espín, "Modelado dinámico y control de maniobras de dispositivos submarinos," 2015.

[26] M. P. De La Portilla et al. "Modelado Dinámico y Control de un Dispositivo Sumergido Provisto de Actuadores Hidrostáticos," RIAI - Rev. Iberoam. Autom. e Inform. Ind., vol. 15, no. 1, pp. 12-23, 2018, doi: 10.4995/riai.2017.8824.

[27] A. J. Sørensen, Marine Control Systems Propulsion and Motion Control of Ships and Ocean Structures. 2013.

[28] "Páginas - $\quad$ Estadística Mensual." http://www.puertos.es/es-es/estadisticas/Pagina s/estadistica_mensual.asp(acc. May 11, 2018).

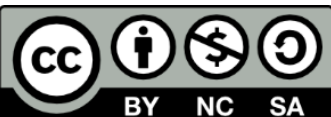

(C) 2021 by the authors. Submitted for possible open access publication under the terms and conditions of the Creative Commons Attribution CC BY-NC-SA 4.0 license (https://creativecommons.org/licenses/byncsa/4.0/deed.es). 